\title{
Recurring Mean Inequality of Random Variables
}

\author{
Mingjin Wang \\ Department of Applied Mathematics, Jiangsu Polytechnic University, Changzhou, Jiangsu 213164, China
}

Correspondence should be addressed to Mingjin Wang, wang197913@126.com

Received 16 August 2007; Revised 25 February 2008; Accepted 9 May 2008

Recommended by Jewgeni Dshalalow

A multidimensional recurring mean inequality is shown. Furthermore, we prove some new inequalities, which can be considered to be the extensions of those established inequalities, including, for example, the Polya-Szegö and Kantorovich inequalities .

Copyright (c) 2008 Mingjin Wang. This is an open access article distributed under the Creative Commons Attribution License, which permits unrestricted use, distribution, and reproduction in any medium, provided the original work is properly cited.

\section{Introduction}

The theory of means and their inequalities is fundamental to many fields including mathematics, statistics, physics, and economics.This is certainly true in the area of probability and statistics. There are large amounts of work available in the literature. For example, some useful results have been given by Shaked and Tong [1], Shaked and Shanthikumar [2], Shaked et al. [3], and Tong [4,5]. Motivated by different concerns, there are numerous ways to introduce mean values. In probability and statistics, the most commonly used mean is expectation. In [6], the author proves the mean inequality of two random variables. The purpose of the present paper is to establish a recurring mean inequality, which generalizes the mean inequality of two random variables to $n$ random variables. This result can, in turn, be extended to establish other new inequalities, which include generalizations of the Polya-Szegö and Kantorovich inequalities [7].

We begin by introducing some preliminary concepts and known results which can also be found in [6].

Definition 1.1. The supremum and infimum of the random variable $\xi$ are defined as $\inf _{x}\{x: P(\xi \leq$ $x)=1\}$ and $\sup _{x}\{x: P(\xi \geq x)=1\}$, respectively, and denoted by sup $\xi$ and inf $\xi$.

Definition 1.2. If $\xi$ is bounded, the arithmetic mean of the random variable $\xi, A(\xi)$, is given by

$$
A(\xi)=\frac{\sup \xi+\inf \xi}{2} .
$$


In addition, if inf $\xi \geq 0$, one defines the geometric mean of the random variable $\xi, G(\xi)$, to be

$$
G(\xi)=\sqrt{\sup \xi \cdot \inf \xi}
$$

Definition 1.3. If $\xi_{1}, \ldots, \xi_{n}$ are bounded random variables, the independent arithmetic mean of the product of random variables $\xi_{1}, \ldots, \xi_{n}, \bar{A}\left(\xi_{1}, \ldots, \xi_{n}\right)$ is given by

$$
\bar{A}\left(\xi_{1}, \ldots, \xi_{n}\right)=\frac{\prod_{i=1}^{n} \sup \xi_{i}+\prod_{i=1}^{n} \inf \xi}{2} .
$$

Definition 1.4. If $\xi_{1}, \ldots, \xi_{n}$ are bounded random variables with inf $\xi_{i} \geq 0, i=1, \ldots, n$, one defines the independent geometric mean of the product of random variables $\xi_{1}, \ldots, \xi_{n}$ to be

$$
\bar{G}\left(\xi_{1}, \ldots, \xi_{n}\right)=\sqrt{\prod_{i=1}^{n} \sup \xi_{i} \inf \xi_{i} .}
$$

Remark 1.5. If $\xi_{1}, \ldots, \xi_{n}$ are independent, then

$$
\begin{aligned}
& \bar{A}\left(\xi_{1}, \ldots, \xi_{n}\right)=A\left(\prod_{i=1}^{n} \xi_{i}\right), \\
& \bar{G}\left(\xi_{1}, \ldots, \xi_{n}\right)=G\left(\prod_{i=1}^{n} \xi_{i}\right) .
\end{aligned}
$$

The mean inequality of two random variables [6].

Theorem 1.6. Let $\xi$ and $\eta$ be bounded random variables. If inf $\xi>0$ and $\inf \eta>0$, then

$$
\frac{E \xi^{2} \cdot E \eta^{2}}{E^{2}(\xi \eta)} \leq \frac{\bar{A}^{2}(\xi, \eta)}{\bar{G}^{2}(\xi, \eta)}
$$

Equality holds if and only if

$$
\begin{gathered}
P\left\{\left(\frac{\xi}{\eta}=\frac{a}{B}\right) \cup\left(\frac{\xi}{\eta}=\frac{A}{b}\right)\right\}=1, \\
G\left(\eta^{2}\right) E \xi^{2}=G\left(\xi^{2}\right) E \eta^{2}
\end{gathered}
$$

for $A=\sup \xi, B=\sup \eta, a=\inf \xi, b=\inf \eta$.

\section{Main results}

Our main results are given by the following theorem.

Theorem 2.1. Suppose that $\xi_{1}, \ldots, \xi_{n}, \xi_{n+1}$ are bounded random variables, inf $\xi_{i}>0, i=1, \ldots, n+1$. Let $\{U(n)\}$ be a sequence of real numbers. If

$$
\frac{\prod_{i=1}^{n} E \xi_{i}^{2}}{E^{2}\left(\prod_{i=1}^{n} \xi_{i}\right)} \leq U(n),
$$

then

$$
\frac{\prod_{i=1}^{n+1} E \xi_{i}^{2}}{E^{2}\left(\prod_{i=1}^{n+1} \xi_{i}\right)} \leq \frac{\bar{A}^{2}\left(\xi_{1}, \ldots, \xi_{n+1}\right)}{\bar{G}^{2}\left(\xi_{1}, \ldots, \xi_{n+1}\right)} U(n)
$$


Proof. Let $A_{i}=\sup \xi_{i}, a_{i}=\inf \xi_{i}, i=1, \ldots, n+1$. We have

$$
P\left\{\left(\xi_{1} \cdots \xi_{n} A_{n+1}-a_{1} \cdots a_{n} \xi_{n+1}\right)\left(A_{1} \cdots A_{n} \xi_{n+1}-\xi_{1} \cdots \xi_{n} a_{n+1}\right) \geq 0\right\}=1 .
$$

So

$$
P\left\{\left(A_{1} \cdots A_{n+1}+a_{1} \cdots a_{n+1}\right) \xi_{1} \cdots \xi_{n+1} \geq A_{1} a_{1} \cdots A_{n} a_{n} \xi_{n+1}^{2}+A_{n+1} a_{n+1} \xi_{1}^{2} \cdots \xi_{n}^{2}\right\}=1,
$$

which implies that

$$
\left(A_{1} \cdots A_{n+1}+a_{1} \cdots a_{n+1}\right) E\left(\xi_{1} \cdots \xi_{n+1}\right) \geq A_{1} a_{1} \cdots A_{n} a_{n} E\left(\xi_{n+1}^{2}\right)+A_{n+1} a_{n+1} E\left(\xi_{1}^{2} \cdots \xi_{n}^{2}\right) .
$$

Using the Jensen inequality [7] and assumption (2.1), we get

$$
\begin{aligned}
\left(A_{1} \cdots A_{n+1}+a_{1} \cdots a_{n+1}\right) E\left(\xi_{1} \cdots \xi_{n+1}\right) & \geq A_{1} a_{1} \cdots A_{n} a_{n} E\left(\xi_{n+1}^{2}\right)+A_{n+1} a_{n+1} E^{2}\left(\xi_{1} \cdots \xi_{n}\right) \\
& \geq A_{1} a_{1} \cdots A_{n} a_{n} E\left(\xi_{n+1}^{2}\right)+A_{n+1} a_{n+1} \frac{E \xi_{1}^{2} \cdots E \xi_{n}^{2}}{U(n)} \\
& \geq 2\left[A_{1} a_{1} \cdots A_{n} a_{n} E\left(\xi_{n+1}^{2}\right) A_{n+1} a_{n+1} \frac{E \xi_{1}^{2} \cdots E \xi_{n}^{2}}{U(n)}\right]^{1 / 2} .
\end{aligned}
$$

Hence,

$$
\left[\frac{\bar{G}^{2}\left(\xi_{1}, \ldots, \xi_{n+1}\right) E \xi_{1}^{2} \cdots E \xi_{n+1}^{2}}{U(n)}\right]^{1 / 2} \leq \bar{A}\left(\xi_{1}, \ldots, \xi_{n+1}\right) E\left(\xi_{1} \cdots \xi_{n+1}\right),
$$

from which (2.2) follows. immediate.

Combining this result with Theorem 1.6, the following recurring inequalities are Corollary 2.2. Let $\xi_{1}, \ldots, \xi_{n}$ be bounded random variables. If inf $\xi_{i}>0, i=1, \ldots, n$, then

$$
\begin{aligned}
\frac{E \xi_{1}^{2} E \xi_{2}^{2}}{E^{2}\left(\xi_{1} \xi_{2}\right)} & \leq \frac{\bar{A}^{2}\left(\xi_{1}, \xi_{2}\right)}{\bar{G}^{2}\left(\xi_{1}, \xi_{2}\right)} \\
\frac{E \xi_{1}^{2} E \xi_{2}^{2} E \xi_{3}^{2}}{E^{2}\left(\xi_{1} \xi_{2} \xi_{3}\right)} & \leq \frac{\bar{A}^{2}\left(\xi_{1}, \xi_{2}, \xi_{3}\right)}{\bar{G}^{2}\left(\xi_{1}, \xi_{2}, \xi_{3}\right)} \frac{\bar{A}^{2}\left(\xi_{1}, \xi_{2}\right)}{\bar{G}^{2}\left(\xi_{1}, \xi_{2}\right)} \\
\vdots & \\
\frac{\prod_{k=1}^{n} E \xi_{k}^{2}}{E^{2}\left(\prod_{k=1}^{n} \xi_{k}\right)} & \leq \prod_{k=2}^{n} \frac{\bar{A}^{2}\left(\xi_{1}, \ldots \xi_{k}\right)}{\bar{G}^{2}\left(\xi_{1}, \ldots \xi_{k}\right)} .
\end{aligned}
$$




\section{Some applications}

In this section, we exhibit some of the applications of the inequalities just obtained. We make use of the following known lemma which we state here without proof.

Lemma 3.1. If $0<m_{2} \leq m_{1} \leq M_{1} \leq M_{2}$, then

$$
\frac{(1 / 2)\left(m_{1}+M_{1}\right)}{\sqrt{m_{1} M_{1}}} \leq \frac{(1 / 2)\left(m_{2}+M_{2}\right)}{\sqrt{m_{2} M_{2}}} .
$$

Theorem 3.2 (the extensions of the inequality of Polya-Szegö). Let $a_{i j}>0, a_{i}=\min _{j} a_{i j}, A_{i}=$ $\max _{j} a_{i j}$, for $i=1, \ldots, n$ and $j=1, \ldots, m$. Then,

$$
\prod_{i=1}^{n} \sum_{j=1}^{m} a_{i j}^{2} \leq \frac{m^{n-2}}{4^{n-1}} \prod_{k=2}^{n} \frac{\left(a_{1} \cdots a_{k}+A_{1} \cdots A_{k}\right)^{2}}{a_{1} \cdots a_{k} A_{1} \cdots A_{k}}\left(\sum_{j=1}^{m} \prod_{i=1}^{n} a_{i j}\right)^{2} .
$$

Proof. This result is a consequence of inequality (2.8). Let $\xi_{1}$ have the distribution

$$
P\left(\xi_{1}=a_{1 j}\right)=\frac{1}{m}, \quad j=1, \ldots, m .
$$

We define $n-1$ functions as follows:

$$
f_{i}\left(a_{1 j}\right)=a_{i j}, \quad i=2, \ldots, n, j=1, \ldots, m
$$

Let $\xi_{i}=f_{i}\left(\xi_{1}\right), i=2, \ldots, n$. Then,

$$
\begin{gathered}
E \xi_{i}^{2}=\frac{1}{m} \sum_{j=1}^{m} a_{i j}^{2}, \quad i=1, \ldots, n, \\
E\left(\xi_{1} \cdots \xi_{n}\right)=\frac{1}{m} \sum_{j=1}^{m} \prod_{i=1}^{n} a_{i j} \\
\bar{A}\left(\xi_{1}, \ldots, \xi_{k}\right)=\frac{1}{2}\left(a_{1} \cdots a_{k}+A_{1} \cdots A_{k}\right), \quad \bar{G}\left(\xi_{1}, \ldots, \xi_{k}\right)=\sqrt{a_{1} \cdots a_{k} A_{1} \cdots A_{k}} .
\end{gathered}
$$

Inequality (2.8) then becomes

$$
\frac{\prod_{i=1}^{n}(1 / m) \sum_{j=1}^{m} a_{i j}^{2}}{\left((1 / m) \sum_{j=1}^{m} \prod_{i=1}^{n} a_{i j}\right)^{2}} \leq \prod_{k=2}^{n} \frac{\left[(1 / 2)\left(a_{1} \cdots a_{k}+A_{1} \cdots A_{k}\right)\right]^{2}}{\left[\sqrt{a_{1} \cdots a_{k} A_{1} \cdots A_{k}}\right]^{2}},
$$

from which our result follows.

Remark 3.3. For $n=2$, we can get the inequality of Polya-Szegö [7]:

$$
\left(\sum_{k=1}^{m} a_{k}^{2}\right)\left(\sum_{k=1}^{m} b_{k}^{2}\right) \leq \frac{1}{4}\left(\sqrt{\frac{A B}{a b}}+\sqrt{\frac{a b}{A B}}\right)^{2}\left(\sum_{k=1}^{m} a_{k} b_{k}\right)^{2},
$$

where $a_{k}, b_{k}>0, k=1, \ldots, m, a=\min a_{k}, A=\max a_{k}, b=\min b_{k}$, and $B=\max b_{k}$. 
Theorem 3.4 (the extensions of Kantorovich's inequality). Let $A$ be an $m \times m$ positive Hermitian matrix. Denote by $\lambda_{1}$ and $\lambda_{m}$ the maximum and minimum eigenvalues of $A$, respectively. For real $\beta_{1}, \ldots, \beta_{n}$ and $\beta=\beta_{1}+\cdots+\beta_{n}$, and any vector $0 \neq x \in \mathbb{R}^{m}$, the following inequality is satisfied:

$$
\frac{\prod_{i=1}^{n} x^{*} A^{\beta_{i}} x}{\left(x^{*} A^{\beta / 2} x\right)^{2}} \leq \frac{\left(x^{*} x\right)^{n-2}}{4^{n-1}} \prod_{k=2}^{n} \frac{\left[l_{1} \cdots l_{k}+L_{1} \cdots L_{k}\right]^{2}}{l_{1} \cdots l_{k} L_{1} \cdots L_{k}}
$$

where

$$
l_{i}=\left\{\begin{array}{ll}
\lambda_{m}^{\beta_{i} / 2}, & \beta_{i} \geq 0, \\
\lambda_{1}^{\beta_{i} / 2}, & \beta_{i}<0,
\end{array} \quad L_{i}=\left\{\begin{array}{ll}
\lambda_{1}^{\beta_{i} / 2}, & \beta_{i} \geq 0, \\
\lambda_{m}^{\beta_{i} / 2}, & \beta_{i}<0,
\end{array} \quad i=1, \ldots, n .\right.\right.
$$

Proof. Let $\lambda_{1} \geq \cdots \geq \lambda_{m}$ be eigenvalues of $A$ and let $\Lambda=\operatorname{diag}\left(\lambda_{1}, \ldots, \lambda_{m}\right)$. There is a Hermitian matrix $U$ that satisfies

$$
A=U^{*} \Lambda U
$$

Let

$$
y=U x=\left(y_{1}, y_{2}, \ldots, y_{m}\right)^{T}, \quad p_{i}=\frac{\left|y_{i}\right|^{2}}{\sum_{i=1}^{m}\left|y_{i}\right|^{2}}, \quad i=1, \ldots, m .
$$

Then,

$$
\begin{aligned}
\frac{\prod_{i=1}^{n} x^{*} A^{\beta_{i}} x}{\left(x^{*} A^{\beta / 2} x\right)^{2}} & =\frac{\prod_{i=1}^{n} x^{*} U^{*} \Lambda^{\beta_{i}} U x}{\left(x^{*} U^{*} \Lambda^{\beta / 2} U x\right)^{2}} \\
& =\frac{\prod_{i=1}^{n} y^{*} \Lambda^{\beta_{i}} y}{\left(y^{*} \Lambda^{\beta / 2} y\right)^{2}} \\
& =\frac{\left(y^{*} y\right)^{n-2} \prod_{i=1}^{n} \sum_{k=1}^{m} \lambda_{k}^{\beta_{i}} p_{k}}{\left(\sum_{k=1}^{m} \lambda_{k}^{\beta / 2} p_{k}\right)^{2}} \\
& =\frac{\left(x^{*} x\right)^{n-2} \prod_{i=1}^{n} \sum_{k=1}^{m} \lambda_{k}^{\beta_{i}} p_{k}}{\left(\sum_{k=1}^{m} \lambda_{k}^{\beta / 2} p_{k}\right)^{2}} .
\end{aligned}
$$

What remains to show is that

$$
\frac{\prod_{i=1}^{n} \sum_{k=1}^{m} \lambda_{k}^{\beta_{i}} p_{k}}{\left(\sum_{k=1}^{m} \lambda_{k}^{\beta / 2} p_{k}\right)^{2}} \leq \frac{1}{4^{n-1}} \prod_{k=2}^{n} \frac{\left[l_{1} \cdots l_{k}+L_{1} \cdots L_{k}\right]^{2}}{l_{1} \cdots l_{k} L_{1} \cdots L_{k}}, \quad \forall p_{i} \geq 0, \sum_{i=1}^{m} p_{i}=1
$$

We define the random variable $\zeta$, and assign $P\left(\zeta=\lambda_{i}\right)=p_{i}, i=1, \ldots, m$. Suppose $\xi_{i}=$ $\zeta^{\beta_{i} / 2}, i=1, \ldots, n$. Notice that $\lambda_{1}$ and $\lambda_{n}$ are the upper and lower bounds of the random variable $\zeta$, so $l_{i}$ and $L_{i}$ are the lower and upper bounds of $\xi_{i}$. According to Lemma 3.1, we know that

$$
\frac{\bar{A}^{2}\left(\xi_{1}, \ldots, \xi_{k}\right)}{\bar{G}^{2}\left(\xi_{1}, \ldots, \xi_{k}\right)} \leq \frac{\left[(1 / 2)\left(l_{1} \cdots l_{k}+L_{1} \cdots L_{k}\right)\right]^{2}}{\left[\sqrt{l_{1} \cdots l_{k} L_{1} \cdots L_{k}}\right]^{2}}
$$


Noticing that

$$
E\left(\xi_{1} \cdots \xi_{n}\right)=E \zeta^{\beta / 2}=\sum_{k=1}^{m} \lambda_{k}^{\beta / 2} p_{k}
$$

we can use inequality (2.8) to express inequality (3.13) as

$$
\frac{E \xi_{1}^{2} \cdots E \xi_{n}^{2}}{E^{2}\left(\xi_{1} \cdots \xi_{n}\right)} \leq \prod_{k=2}^{n} \frac{\left[(1 / 2)\left(l_{1} \cdots l_{k}+L_{1} \cdots L_{k}\right)\right]^{2}}{\left[\sqrt{l_{1} \cdots l_{k} L_{1} \cdots L_{k}}\right]^{2}}
$$

Remark 3.5. If $n=2, \beta_{1}=1$, and $\beta_{2}=-1$, this inequality takes the form

$$
\frac{x^{*} A x x^{*} A^{-1} x}{\left(x^{*} x\right)^{2}} \leq \frac{\left(\lambda_{1}+\lambda_{m}\right)^{2}}{4 \lambda_{1} \lambda_{m}}
$$

which is Kantorovich's inequality [7].

\section{References}

[1] M. Shaked and Y. L. Tong, "Inequalities for probability contents of convex sets via geometric average," Journal of Multivariate Analysis, vol. 24, no. 2, pp. 330-340, 1988.

[2] M. Shaked and J. G. Shanthikumar, Stochastic Orders and Their Applications, Probability and Mathematical Statistics, Academic Press, Boston, Mass, USA, 1994.

[3] M. Shaked, J. G. Shanthikumar, and Y. L. Tong, "Parametric Schur convexity and arrangement monotonicity properties of partial sums," Journal of Multivariate Analysis, vol. 53, no. 2, pp. 293-310, 1995.

[4] Y. L. Tong, "Some recent developments on majorization inequalities in probability and statistics," Linear Algebra and Its Applications, vol. 199, supplement 1, pp. 69-90, 1994.

[5] Y. L. Tong, "Relationship between stochastic inequalities and some classical mathematical inequalities," Journal of Inequalities and Applications, vol. 1, no. 1, pp. 85-98, 1997.

[6] M. Wang, "The mean inequality of random variables," Mathematical Inequalities \& Applications, vol. 5, no. 4, pp. 755-763, 2002.

[7] G. H. Hardy, J. E. Littlewood, and G. Pólya, Inequalities, Cambridge University Press, Cambridge, UK, 2nd edition, 1952. 